\title{
Optimization Design of Thermoelectric Power Generation System of Waste Heat Recovery
}

\author{
Xiaoyu Cheng ${ }^{1,2}$, Huiqing $\mathrm{Hu}^{1}$, Yunqing Bai ${ }^{1}$, Gang $\mathrm{Xu}^{1}$, Chongju $\mathrm{Hu}^{1}$, Yukun $\mathrm{Li}^{1,2}$, and Zhizhong Jiang ${ }^{1, *}$ \\ ${ }^{1}$ Key Laboratory of Neutronics and Radiation Safety, Institute of Nuclear Energy Safety Technology, Chinese Academy of Sciences, \\ Hefei Anhui, 230031, China \\ ${ }^{2}$ University of Science and Technology of China, Hefei Anhui, 230027, China
}

\begin{abstract}
Considering that many parameters with mutual influence which were difficult to be considered comprehensively were involved in the optimization design of thermoelectric power generation system, a method that can calculate the optimal design scheme quantitatively according to the heat source characteristic was proposed in this work. The optimum working temperature of the thermoelectric module was determined by coupling the measured thermoelectric module performance curve with the temperature relationship of the cold and hot ends under the design condition. Then, based on the iterative method, the influence of various fin radiator structural parameters on heat dissipation performance was quantitatively analyzed by FLUENT software, so as to deliver the best combination of radiator parameters. The results showed that under the condition of constant heat flux density of $36 \mathrm{kw} / \mathrm{m}^{2}$, in order to obtain the best system efficiency, the cold end temperature should be controlled as $50{ }^{\circ} \mathrm{C}$, and the fin radiator base thickness, fin spacing and fin height of the matched radiator should be $8 \mathrm{~mm}, 3 \mathrm{~mm}$ and $90 \mathrm{~mm}$ respectively.
\end{abstract}

\section{Introduction}

Thermoelectric power generation device of semiconductor (TEG) is a device that can directly convert thermal energy into electrical energy, with the advantages of no pollution, compact structure, no rotating parts, no noise and no maintenance ${ }^{[1-2]}$. TEG has a broad application prospect in the field of building energy saving. Solar energy around the building and waste heat from heating systems, large appliances and industrial production can all be converted into electrical energy by TEG. Recycling such low-grade heat energy can effectively improve energy utilization rate and plays an important role in saving energy and reducing carbon dioxide emissions ${ }^{[3]}$.

However, the thermoelectric conversion efficiency of TEG in practical application is generally low, which seriously restricts its wide popularity in the field of waste heat recovery ${ }^{[4]}$. In order to improve its thermoelectric conversion efficiency, thermoelectric materials were mainly emphasized in previous studies, such as the adoption of nanotechnology and band regulation, and the figure of merit (ZT) of thermoelectric power generation materials has more than doubled in the past ten years ${ }^{[5-8]}$. In addition, as for the application of thermoelectric power generation technology, it is necessary to carry out optimization design research of TEG system.

The optimization of TEG system depends on its heat source environment. The research shows that under the condition of constant heat source temperature, enhancing the heat dissipation capability can effectively reduce the cold end temperature of the power generation module and improve the thermoelectric conversion efficiency of the system; within the appropriate temperature range, the power generation module has the best output performance ${ }^{[9-12]}$. However, under the condition of nonconstant heat source temperature, the change of the heat dissipation capability of the cold end will lead the temperature of both ends of thermoelectric element to change accordingly, and at the same time, the thermoelectric figure of merit (ZT) and heat conductivity coefficient $\lambda$ of the thermoelectric element of semiconductor will change greatly according to different cold and hot end temperature, which makes the determination of optimum operating temperature of generator module become very complex ${ }^{[13]}$.

In terms of heat dissipation design, plate-fin radiator is mostly chosen in TEG system to improve heat dissipation performance through the optimization of shape structure, heat dissipation area, and wind speed and other factors. Due to too many parameters to be considered, the influence rule of a single factor on heat dissipation performance was only qualitatively analyzed in previous studies, which was used as the basis for radiator design, and the optimal parameter combination was not quantitatively calculated ${ }^{[14-16]}$. Therefore, the comprehensive consideration of various design parameters and further optimization of radiator model selection are of great significance for improving the actual output performance and heat utilization efficiency of the power generation unit.

\footnotetext{
* Zhizhong Jiang: zhizhong.jiang@fds.org.cn
} 
The differential equation of heat conduction is used to simulate the temperature distribution in thermoelectric element with $\mathrm{Bi}_{2} \mathrm{Te}_{3}$ as the base, and FLUENT software is used to simulate the fin heat dissipation, which is a common simulating calculation method. The rationality of the process and accuracy of the results have been fully demonstrated in previous studies ${ }^{[16-21]}$. Based on these, an optimal design scheme of the TEG system was calculated by means of coupling and iterative calculation in this paper, so as to provide guidance for the practical engineering application of TEG.

\section{TEG System Modeling}

\subsection{Initial conditions and design objective}

The environment temperature is $20^{\circ} \mathrm{C}$, and the heat flux density of waste heat is about $36 \mathrm{~kW} / \mathrm{m}^{2}$. The design objective is to achieve the best thermoelectric performance of the thermoelectric element considering the operating economy of the heat dissipation module.

\subsection{System module}

The overall structure of TEG is composed of the power generator module and the heat dissipation module, as shown in Figure 1. Heat dissipation module includes radiator and fan, among which, radiator is adopted with plate-fin structure, with the characteristics of uniform distribution and simple form, which is beneficial to industrial mass production and application. It is assumed that the thermal properties and heat flux density of each unit are the same when working, and it is regarded as a whole.

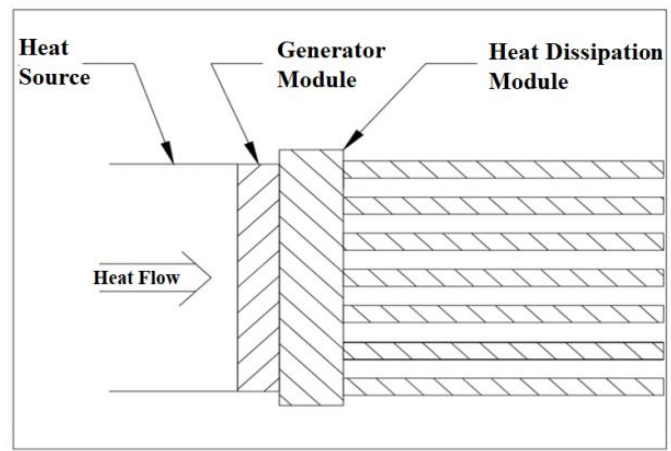

Fig. 1. Structure diagram of the thermoelectric power generation system

\subsection{Specifications and parameters of generator module}

The semiconductor material selected for thermoelectric element is ternary solid solution alloy with $\mathrm{Bi}_{2} \mathrm{Te}_{3}$ as the base; P-type composition is (70\% $75 \%) \mathrm{Sb}_{2} \mathrm{Te}_{3}+(25 \%$ $\sim 30 \%) \mathrm{Bi}_{2} \mathrm{Te}_{3}+\mathrm{P}$-type impurity, and N-type composition is $(90 \% \sim 93 \%) \mathrm{Bi}_{2} \mathrm{Te}_{3}+(7 \% \sim 10 \%) \mathrm{Bi}_{2} \mathrm{Se}_{3}+\mathrm{N}-$ type impurity. According to the actual structure parameters of the thermoelectric element, the established model size of the thermoelectric element is $40 \mathrm{~mm} \mathrm{x} 40$ $\mathrm{mm}$. The relationship curve between the measured thermoelectric conversion efficiency and the temperature of the cold and hot ends is shown in Figure $2^{[22]}$.

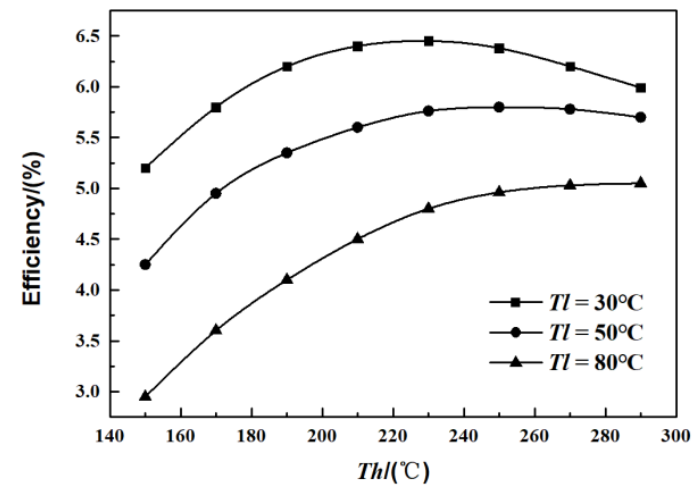

Fig. 2. The measured thermoelectric conversion efficiency curve of the thermoelectric element ( $T_{h}$ and $T_{l}$ represent temperature of the hot end and cold end)

On the whole, as the cold end temperature drops from $80{ }^{\circ} \mathrm{C}$ to $30{ }^{\circ} \mathrm{C}$, the thermoelectric conversion efficiency gradually increases. For a constant cold end temperature, as the hot end temperature increases, the thermoelectric conversion efficiency first increases, then tends to be steady, and finally decreases, and there is an optimal temperature range.

\section{Thermoelectric Characteristics Ana- lysis of Power Generation Module}

Because the thermoelectric optimization value (ZT) and heat conductivity coefficient $\lambda$ of the thermoelectric power generation element of semiconductor will change according to different cold and hot ends temperature, the actual thermoelectric conversion efficiency also changes accordingly. Under the condition of constant heat flux density of heat source, controlling the cold and hot ends in the optimal temperature range is one of the important ways to improve the thermoelectric conversion efficiency.

When the heat flow gets through the generator module, it involves the Seebeck effect, the Peltier effect, the Joule effect and the Thomson effect in the interior. If it is assumed that the material of the thermoelectric element is homogeneous, the heat is transmitted only along the direction of the current, and the contact resistance of the cold and hot ends is ignored, the differential equation of heat conduction in the thermoelectric element in the interior can be expressed as [17-19].

$$
\begin{aligned}
& \lambda_{n} \frac{\partial^{2} T_{n}}{\partial x^{2}}-\beta_{n} I \frac{\partial T_{n}}{\partial x}+\frac{R_{n} I^{2}}{d_{n}}=0 \\
& \lambda_{p} \frac{\partial^{2} T_{p}}{\partial x^{2}}-\beta_{p} I \frac{\partial T_{p}}{\partial x}+\frac{R_{p} I^{2}}{d_{p}}=0
\end{aligned}
$$

Where $\lambda_{n}$ and $\lambda p$ represent the heat conductivity coefficients, $\mathrm{W} /\left(\mathrm{m} \cdot{ }^{\circ} \mathrm{C}\right) ; T_{n}$ and $T_{p}$ represent temperature, $\mathrm{K} ; \beta_{n}$ and $\beta_{p}$ represent the Thomson coefficients; $R_{n}$ and $R_{p}$ represent resistance, $\Omega ; d_{n}$ and $d_{p}$ represent length, m; $I$ represents the output current, A.

In addition, the following basic equations are also used ${ }^{[17]}$. 


$$
\begin{gathered}
\alpha=\alpha_{p}-\alpha_{n} \\
U=\int_{T_{l}}^{T_{h}} \alpha(T) d T \\
R=R_{n}+R_{p}
\end{gathered}
$$

Where $\alpha$ represents Seebeck coefficient, V/K; $U$ represents the output voltage, $\mathrm{V} ; T_{h}$ and $T_{l}$ represent the temperature of hot and cold ends of the power generation film, $\mathrm{K} ; \quad R$ represents the total resistance of thermoelectric element, $\Omega$.

If it is considered that half of the joule heat generated in the thermoelectric element is transferred to the hot end and the other half to the cold end, the heat flux density at the cold and hot ends can be approximately expressed as $[17,18]$ :

$$
\begin{gathered}
q_{h}=\alpha T_{h} I+\lambda \frac{T_{h}-T_{l}}{d}-\frac{R}{2} I^{2} \\
q_{l}=\alpha T_{l} I+\lambda \frac{T_{h}-T_{l}}{d}+\frac{R}{2} I^{2}
\end{gathered}
$$

Where $q_{h}$ and $q_{l}$ represent the heat flux density of the hot and the cold ends of the power generation film, $\mathrm{W} / \mathrm{m}^{2}$.

BAI Zhongkai and others selected the same type of thermoelectric element and obtained the relationship between the heat conductivity coefficient of the thermoelectric element and the temperature change by measuring the diffusion coefficient and the corresponding heat capacity ${ }^{[16]}$ :

$$
\lambda=A+B \times(T+273)+C \times(T+273)^{2}
$$

Where $A=5.7631 ; B=-0.02416 ; C=$ 0.00003593; $T$ represents the average temperature of the cold and hot ends of the power generation film, ${ }^{\circ} \mathrm{C}$.

Combined with the measured thermoelectric conversion efficiency curve of the thermoelectric element (Figure 2), the curve of the relation between the cold and hot ends temperature and thermoelectric conversion efficiency of the thermoelectric element under the design condition is shown in Figure 3.

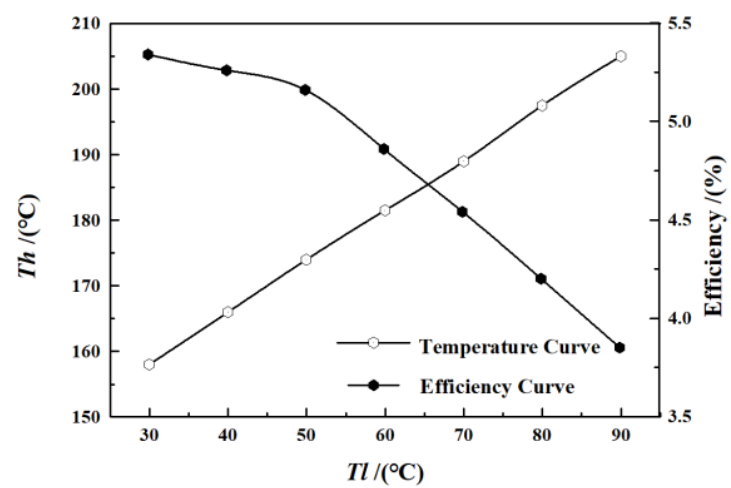

Fig. 3. The curve of the relation between the cold and hot ends temperature and efficiency of thermoelectric element under the design condition

As can be seen, as the temperature of the cold end decreases, the temperature of the hot end also decreases, and the corresponding thermoelectric conversion efficiency gradually increases. When the temperature of the cold end drops to $50{ }^{\circ} \mathrm{C}$, the efficiency reaches $5.16 \%$. When the temperature of the cold end continues to decrease, the growth of conversion efficiency slows down. If the temperature of the cold end further decreases, the additional increase in cooling fan power is larger. Based on the above considerations, the temperature of the cold end is suggested to be controlled at $50{ }^{\circ} \mathrm{C}$. At this time, the electrical power density of TEG is $1.86 \mathrm{~W} / \mathrm{cm}^{2}$, and the heat flux density of the heat dissipation module is $34.14 \mathrm{~W} / \mathrm{cm}^{2}$.

\section{Optimization Design of Heat Dissipa- tion Module}

Radiator is the key module of TEG, the reasonable design of radiator, not only with light weight and small volume, but also with better heat dissipation performance. The performance state is mainly related to the fin parameters, including fin height, fin spacing, base thickness and fin thickness, etc. Based on the heat flux density of $34.14 \mathrm{~kW} / \mathrm{m}^{2}$ and controlling the cold end temperature of the generator module at $50{ }^{\circ} \mathrm{C}$, the iterative method commonly used in heat engineering calculation was proposed to be applied in heat dissipation design in this paper, to evaluate the influence of different fin height, fin spacing and base thickness on the heat dissipation capacity, and obtain the optimal radiator structure based on the minimum required wind speed.

\subsection{Mathematic model}

The overall temperature of radiator is lower than $50{ }^{\circ} \mathrm{C}$, so the influence of heat dissipation through radiation can be ignored, and the heat that flows through radiator sends out to the environment by the means of heat conduction and convection. It is a three-dimensional steady state and incompressible flow heat transfer process in the heat transfer unit, and the k- $\varepsilon$ turbulence model is adopted to reflect turbulent flow on the convection side. The governing equations involved mainly include: thermal diffusion equation, mass conservation equation, momentum conservation equation, energy conservation equation and turbulence $\mathrm{k}-\varepsilon$ model $[16,20,21]$

The thermal diffusion equation is:

$$
\frac{\partial^{2} T}{\partial x^{2}}+\frac{\partial^{2} T}{\partial y^{2}}+\frac{\partial^{2} T}{\partial z^{2}}=0
$$

Where $T$ represents temperature, $\mathrm{K}$.

The mass conservation equation is:

$$
\frac{\partial \rho}{\partial \tau}+\boldsymbol{d i v}(\rho V)=0
$$

Where $\rho$ represents density, $\mathrm{kg} / \mathrm{cm}^{3} ; \tau$ represents time, $\mathrm{s} ; V$ represents the velocity vector, $\mathrm{m} / \mathrm{s} ; u, v$ and $w$ respectively represent the components of the velocity vector in the $\mathrm{x}, \mathrm{y}$ and $\mathrm{z}$ directions, $\mathrm{m} / \mathrm{s}$.

The momentum conservation equation is:

$$
\begin{aligned}
& \frac{\partial(\rho u)}{\partial \tau}+\boldsymbol{d i v}(\rho u V)=\operatorname{div}(\eta \operatorname{grad} u)+F_{x}-\frac{\partial p}{\partial x} \\
& \frac{\partial(\rho v)}{\partial \tau}+\boldsymbol{d i v}(\rho v V)=\operatorname{div}(\eta \operatorname{grad} v)+F_{y}-\frac{\partial p}{\partial y}
\end{aligned}
$$




$$
\frac{\partial(\rho w)}{\partial \tau}+\boldsymbol{d i v}(\rho w V)=\operatorname{div}(\eta \operatorname{grad} w)+F_{z}-\frac{\partial p}{\partial z}
$$

Where $\eta$ represents dynamic viscosity coefficient, $\mathrm{Pa} \cdot \mathrm{s} ; F_{x}, F_{y}$ and $F_{z}$ represent the components of the volume force in the $\mathrm{x}, \mathrm{y}$ and $\mathrm{z}$ directions respectively, and $\mathrm{p}$ represents the pressure on the element body.

The energy conservation equation is:

$$
\frac{\partial(\rho T)}{\partial \tau}+\boldsymbol{d i v}(\rho V T)=\operatorname{div}\left(\frac{\lambda}{c_{p}} \operatorname{grad} T\right)+F_{T}
$$

Where $C_{p}$ represents specific heat capacity, $\mathrm{J} /(\mathrm{kg} \mathrm{K})$; $F_{T}$ represents the heat transferred from mechanical work to fluid flow. is:

The governing equation of turbulent kinetic energy $k$

$$
\frac{\partial(\rho k)}{\partial \tau}+\frac{\partial\left(\rho u_{i} k\right)}{\partial x_{i}}=\frac{\partial}{\partial x_{i}}\left[\left(\eta+\frac{\eta_{t}}{\sigma_{k}}\right) \frac{\partial k}{\partial x_{i}}\right]+G_{k}-\rho \varepsilon
$$

In formula (15):

$$
\begin{gathered}
\eta_{t}=\rho C_{\eta} \frac{k^{2}}{\varepsilon} \\
G_{k}=2 \eta_{t}\left(\frac{\partial u_{i}}{\partial x_{j}}+\frac{\partial u_{j}}{\partial x_{i}}\right) \frac{\partial u_{i}}{\partial x_{j}}
\end{gathered}
$$

Where $\eta_{t}$ represents turbulent kinetic viscosity coefficient, $\mathrm{Pa} \bullet \mathrm{s} ; G_{k}$ represents the generation rate of turbulence volume, $\mathrm{kg} /\left(\mathrm{s}^{3} \cdot \mathrm{m}\right)$.

The governing equation of dissipation rate $\varepsilon$ is: $\frac{\partial(\rho \varepsilon)}{\partial \tau}+\frac{\partial\left(\rho \eta_{i} \varepsilon\right)}{\partial x_{i}}=\frac{\partial}{\partial x_{i}}\left[\left(\eta+\frac{\eta_{t}}{\sigma_{\varepsilon}}\right) \frac{\partial \varepsilon}{\partial x_{i}}\right]+C_{1 \varepsilon} G_{k} \frac{\varepsilon^{2}}{k}-C_{2 \varepsilon} \rho \frac{\varepsilon^{2}}{k}$

Where $C_{1 \varepsilon}=1.44 ; C_{2 \varepsilon}=1.92 ; \sigma_{k}=1 ; \sigma_{\varepsilon}=1.3 ;$ $C_{\eta}=0.09$.

\subsection{Simulation calculation model}

The calculation model of radiator was established by FLUENT software preprocessor gambit, tetrahedral shape was used in grid division, and a dense grid was arranged on the fin heat dissipation surface. The established structure model and divided grid are shown in Figure 4 and Figure 5.

In this work, grid-independent solution test was carried out on the fin area of the simulation calculation model. The model selection of No.1 and No.20 radiators in Table 1 was selected as the test object, and the heat transfer performance of the fin was compared when the size was $0.1 \mathrm{~mm}, 0.2 \mathrm{~mm}, 0.3 \mathrm{~mm}, 0.5 \mathrm{~mm}$ and $0.7 \mathrm{~mm}$. The results showed that the heat flux will shift greatly when the grid size was larger than $0.3 \mathrm{~mm}$. After considering the requirements of calculation accuracy and calculation speed, the grid size was determined as 0.2 $\mathrm{mm}$.

The radiator is adopted with aluminum material, and the speed inlet condition is adopted. The wind direction is horizontal flow along the fin groove, the outlet is set as pressure outlet, and the heat dissipation surface is set as no slip speed mode. Heat flow flows in from the bottom of the base with a size of $34.14 \mathrm{~kW} / \mathrm{m}^{2}$ and an environment temperature of $20^{\circ} \mathrm{C}$. Steady-state standard turbulence $\mathrm{k}-\varepsilon$ model is adopted for calculation, SIMPLEC algorithm is selected for speed as well as pressure coupling, and the convergence standard is set by default.

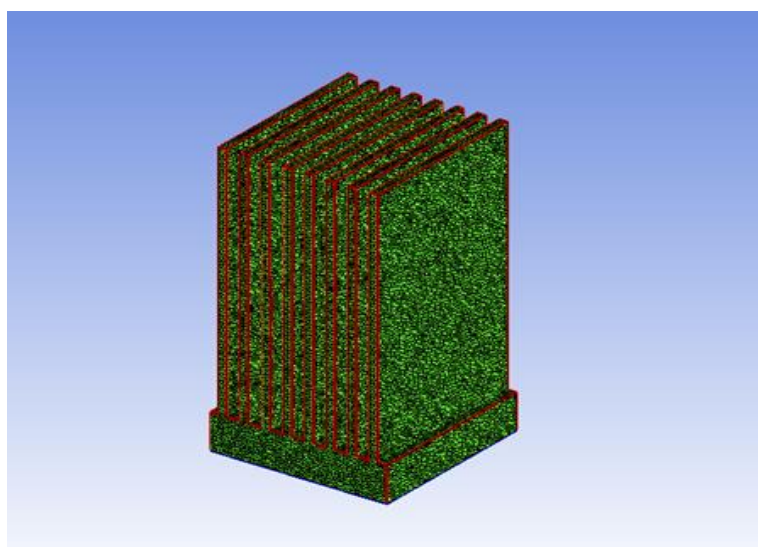

Fig. 4. Schematic diagram of the calculation structure

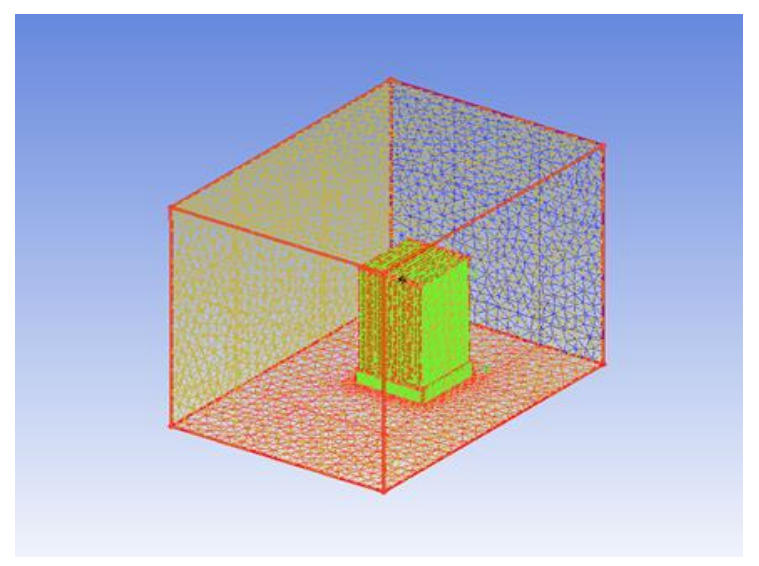

Fig. 5. Overall grid division of the heat dissipation module

\subsection{Radiator structure design}

\subsubsection{Fin height and spacing optimization}

The thickness of the fin shall be $2 \mathrm{~mm}$, and the length and width of the base shall be $45 \mathrm{~mm}$. Accordingly, under the condition that the base thickness is fixed as $10 \mathrm{~mm}, 20$ kinds of radiators with different fin spacing and fin height are given in this paper, as shown in Table 1. The wind speed can be obtained by simulation calculation with the method described in Section 3.1, required by these 20 types of radiators when the average surface temperature under the base is slightly lower than $50{ }^{\circ} \mathrm{C}$, and the result is shown in Figure 6.

Table 1. Type of radiator (a)

( $D$ represent base thickness; $N$ represent number of fin; $S$ represent fin spacing; $H$ represent fin height)

\begin{tabular}{ccccc}
\hline Element & $\boldsymbol{D} /(\mathbf{m m})$ & $\boldsymbol{N}$ & $\boldsymbol{S} /(\mathbf{m m})$ & $\boldsymbol{H} /(\mathbf{m m})$ \\
\hline $\mathbf{1 - 5}$ & 10 & 7 & 4 & $60 / 70 / 80 / 90 / 100$ \\
$\mathbf{6 - 1 0}$ & 10 & 8 & 3.5 & $60 / 70 / 80 / 90 / 100$ \\
$\mathbf{1 1 - 1 5}$ & 10 & 9 & 3 & $60 / 70 / 80 / 90 / 100$ \\
$\mathbf{1 6 - 2 0}$ & 10 & 10 & 2.5 & $60 / 70 / 80 / 90 / 100$ \\
\hline
\end{tabular}




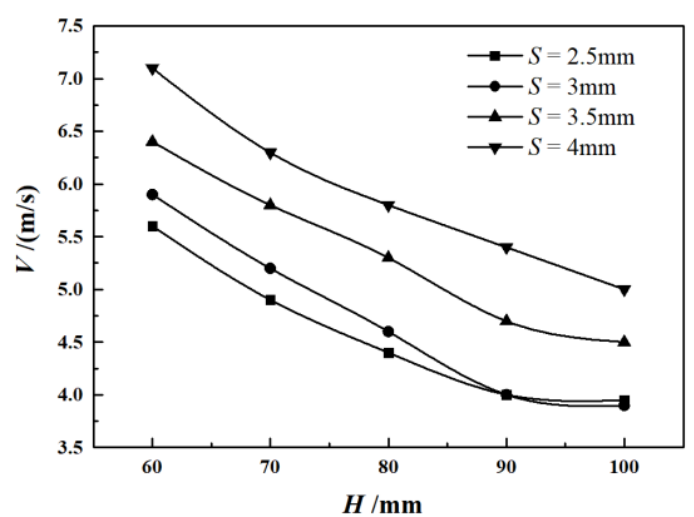

Fig. 6. The relationship between fin spacing/fin height and required wind speed

On the whole, with the increase of fin height and the decrease of spacing, the wind speed required by the radiator gradually decreases, which is due to the increase of heat dissipation area. Particularly, when fin height is $\geq$ $90 \mathrm{~mm}$, the radiator with $2.5 \mathrm{~mm}$ spacing requires wind speed larger than radiator with $3 \mathrm{~mm}$ spacing. It is because as the number of fins increases, on the one hand, the heat dissipation area increases and the heat dissipation is strengthened; on the other hand, the crosssectional area of air circulation decreases, and the effective mass flow decreases, and therefore the heat dissipation is weakened. When the fin height is more than $90 \mathrm{~mm}$ and the fin spacing is less than $3 \mathrm{~mm}$, the amplitude of wind speed decrease slows down or even increases in the opposite direction. Therefore, the fin height and spacing are suitable for $90 \mathrm{~mm}$ and $3 \mathrm{~mm}$.

\subsubsection{Base thickness optimization}

Under the condition that the fin height and spacing are fixed as $90 \mathrm{~mm}$ and $3 \mathrm{~mm}$ respectively, radiators with 7 different base thicknesses are given in this paper, as shown in Table 2. With the same approach, the simulation results are shown in Figure 7.

Table 2. Type of radiator (b)

\begin{tabular}{ccccc}
\hline Element & $\boldsymbol{D} /(\mathbf{m m})$ & $\boldsymbol{N}$ & $\boldsymbol{S} /(\mathbf{m m})$ & $\boldsymbol{H} /(\mathbf{m m})$ \\
& & & & \\
\hline $\mathbf{2 1 - 2 4}$ & $6 / 8 / 10 / 12$ & 9 & 3 & 90 \\
$\mathbf{2 5 - 2 7}$ & $14 / 16 / 18$ & 9 & 3 & 90 \\
\hline
\end{tabular}

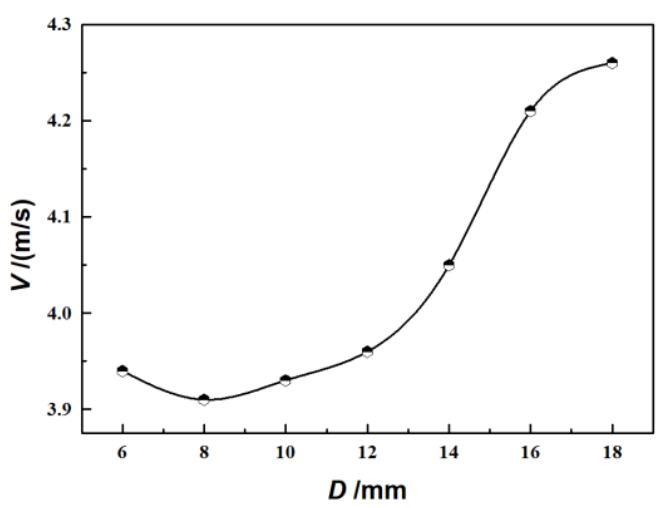

Fig. 7. The relationship between base thickness and required wind speed
If the fin height and spacing parameters are fixed, and when the base is thin, the thermal resistance is small and the heat dissipation performance is improved, it is suitable to choose a smaller fin thickness value. From the figure, when the fin height and fin spacing are fixed as $90 \mathrm{~mm}$ and $3 \mathrm{~mm}$ respectively, the thickness of the base is suitable for $8 \mathrm{~mm}$.

\subsubsection{Iteration optimization}

Under the condition that the base thickness is fixed as 8 $\mathrm{mm}, 8$ kinds of radiators with different fin height and fin spacing are given in this paper, as shown in Table 3. With the same approach, the simulation results are shown in Figure 8.

Table 3. Type of radiator (c)

\begin{tabular}{ccccc}
\hline Element & $\begin{array}{c}\boldsymbol{D} \\
/(\mathbf{m m})\end{array}$ & $\boldsymbol{N}$ & $\boldsymbol{S} /(\mathbf{m m})$ & $\boldsymbol{H} /(\mathbf{m m})$ \\
\hline $\mathbf{2 8 - 3 1}$ & 8 & 9 & 3 & $80 / 90 / 100 / 110$ \\
$\mathbf{3 2 - 3 5}$ & 8 & 10 & 2.5 & $80 / 90 / 100 / 110$ \\
\hline
\end{tabular}

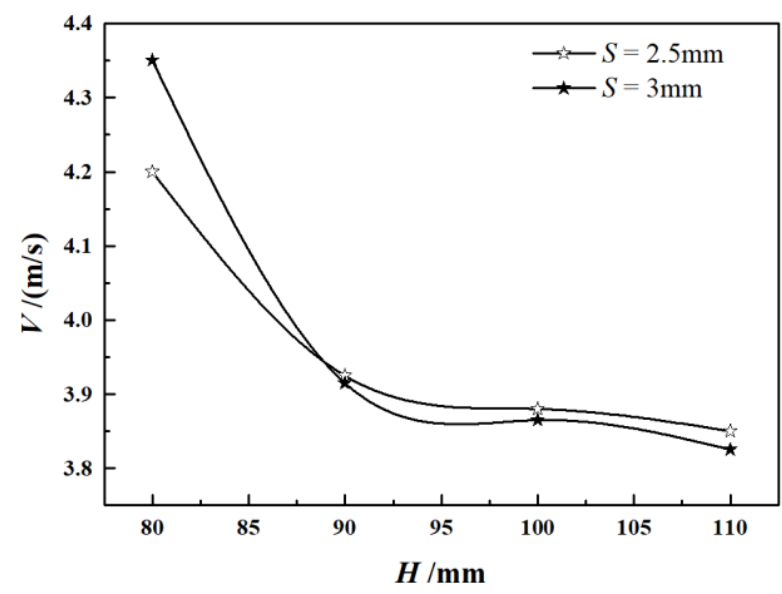

Fig. 8. The relationship between fin spacing/fin height and required wind speed

From the figure, when the base thickness is $8 \mathrm{~mm}$, the optimal values of fin height and spacing are still 90 $\mathrm{mm}$ and $3 \mathrm{~mm}$, and the iteration ends.

In terms of heat dissipation design, 35 different types of fin radiators are listed in Section 4.3. Under the design conditions, the required wind speed varies greatly, with the maximum value 1.9 times of the minimum value. It can be seen that the application of iterative method to find the best radiator structure is of great significance to reduce the mass volume of the device and improve the total efficiency of the system.

\section{Conclusion}

In this paper, the curve of the relation between the thermoelectric conversion efficiency of the generator module and the temperature at both ends is given, and the influence of various structural parameters on the heat dissipation performance is quantitatively analyzed. The specific conclusions are as follows: 
1) Due to the influence of heat source and material characteristics, there is an optimal operating temperature when the thermoelectric element works. Under such a design condition, the temperature of the cold end of the generator module should be controlled at $50{ }^{\circ} \mathrm{C}$. At this time, the efficiency reaches $5.16 \%$ and the system output performance is the best.

2) When the fin thickness is $2 \mathrm{~mm}$, the optimal values of base thickness, fin spacing and fin height are 8 $\mathrm{mm}, 3 \mathrm{~mm}$ and $90 \mathrm{~mm}$ respectively. At this time, when the average surface temperature of the base is slightly lower than $50{ }^{\circ} \mathrm{C}$, the wind speed required is only 3.91 $\mathrm{m} / \mathrm{s}$.

\section{Acknowledgement}

This work was supported by the Youth Program of National Natural Science Foundation of China (No.21805283), the National Key R\&D Program of China (2018YFB1900601).

\section{References}

1. W. Yan, G. Y. Qiu, and X. F. Yuan. Chinese Journal of Power Sources, 40, 8 (2016).

2. J. Y. Zhao, D. S. Zhu, Z. G. Zhou, et al. Chinese Journal of Power Sources, 34, 3 (2010)

3. W, He, S. Wang, X. Zhang, et al. Energy, 91 (2015)

4. R. Y. Nuwayhid, A. Shihadeh, N. Ghaddar. Energy Conversion and Management 46, 9-10 (2005)

5. T. Kajitani, Y. Miyazaki, K. Hayashi, et al. Thermoelectric Energy Conversion and Ceramic Thermoelectrics [C] // Materials Science Forum, 671 (2011)

6. Q. Huang, N. Baluc, Y. Dai, et al. J.Nucl. Mater, 442 (2013)

7. Y. Yang, K. C. Pradel, Q. Jing, et al. Acs Nano, 6, 8 (2012)

8. B. Feng, G. Q. Li, C. C. Zhang, et al. Materials Review, 31 (2017)

9. T. Lin, C. H. Wang, Q. M. Lv, et al. Chinese Journal of Power Sources, 38, 8 (2014)

10. L. Jia, Z. S. Chen, P. HU, et al. Journal of University of Science and Technology of China, 34, 6 (2004)

11. D. Mastbergen. Dissertations \& Theses Grad-works, (2008)

12. C. Yu, K. T. Chau. Energy Conversion \& Management, 50, 6 (2009)

13. Y. Apertet, H. Ouerdane, C. Goupil, et al. Journal of Applied Physics, 116, 14 (2014)

14. S. L. Ma. Optimization and Analysis of Automobile Exhaust Waste Heat Thermo-electric Generation Device [D]. Shenyang University of Technology (2013)

15. D. Astrain, J.G. Vián, A. Martínez, et al. Energy, 35, 2 (2010)
16. Z. K. Bai. Research on Thermal Systems of Semiconductor Thermoelectric Generator in Middlehigh Temperature Waste Heat Recovery [D]. Nanjing University of Aeronautics and Astronautics (2009)

17. L. Guo, F. M. Wang, T. Mi. Chinese Journal of Power Sources, 43, 07 (2019)

18. S. L. Shen, M. L. Tu, Y. Y. Fang, et al. Journal of China Three Gorges University(Natural Sciences), 40, 05 (2018)

19. F. Meng, L. Chen, F. Sun. Energy, 36, 5 (2011)

20. Y. Huang, X. Yu, G. Lu. Journal of Zhejiang University (Engineering Science) 8 (2008)

21. H. K. Cai, Z. J. Weng, Y. D. Liao, et al. Journal of South China University of Technology (Natural Science Edition), 47,1 (2019)

22. Specification for Thermoelectric Power Genera-tion Devices [EB/OL]. http://www. thermonamic. com/TEHP1-1264-0.8-English.pdf. 